\title{
Asthma monitoring in primary care: time to gather more robust evidence
}

See linked article by Turton et al. on pg 28

\author{
*Justin Beilby ${ }^{\mathrm{a}}$ \\ a Executive Dean, Faculty of Health Sciences, The University of \\ Adelaide, Australia \\ *Correspondence: Professor Justin Beilby, Faculty of Health \\ Sciences, The University of Adelaide, South Australia, Australia \\ 5005. Tel: +6188303 5193 Fax: +61 883033788 \\ E-mail: justin.beilby@adelaide.edu.au
}

For people with asthma, the control of symptoms is now the accepted goal of clinical management. ${ }^{1}$ In everyday practice, clinicians use a mixture of clinical measures and an assessment of "the future risk of exacerbations" 1 to determine how aggressive they will be with pharmacological therapy, usually inhaled corticosteroids (ICS). Since there is a lack of correlation between symptoms, resolution of airway inflammation and bronchial hyper-responsiveness, primary care practitioners will often continue therapy with ICS at higher doses than required: if patients are stable, overtreatment is likely to continue, and there is a general reluctance by busy practicing clinicians to decrease the ICS dose at regular intervals.

In this issue of the PCRJ, Turton et al. ${ }^{2}$ have completed an important pilot study exploring the use of point-of-care indirect bronchial hyperresponsiveness (BHR) testing with mannitol in order to monitor and (importantly) adjust the ICS dose. Despite the small numbers, the authors found that there were improvements in Asthma Quality of Life Questionnaire (AQLQ) scores that correlated with improvements in BHR after increases in ICS dosage. Conversely, where the indirect BHR measure was seen to be normal, the general practitioners (GPS) were able to reduce the ICS dose. If indirect BHR testing could predict reliably and consistently the physiological improvements in asthma markers that correlate with improvements in quality of care, and if it could be implemented efficiently, then this measurement tool would be very welcome indeed.

Unfortunately, the evidence for widespread adoption of indirect BHR testing is not conclusive. There have been studies ${ }^{3}$ that have found improved asthma control with the regular use of indirect BHR monitoring, all with increased use of ICS. Conversely, further studies ${ }^{4,5}$ have revealed no real improvements in control. It is currently inappropriate to recommend that there be widespread adoption of a measurement technique that may lead to an increased use of medications such as ICS (with known increased risk of side effects) without clear and consistent evidence of improved outcomes for patients. As Turton et al. comment, ${ }^{2}$ it is time to develop and implement larger trials based in primary care that utilise regular BHR testing to examine the long term health benefits of this monitoring.

Efficient implementation is also required. It is unlikely that busy
GPs will have the time to commit to using this tool on their patients. Implementation will require the training of nursing or allied health staff to interact with patients - as Turton et al. found. ${ }^{2}$ Team-based primary care is the key to successful implementation of this type of ongoing asthma assessment. ${ }^{6}$ In health systems where private practice dominates, a financial incentive may be required. Australia has successfully introduced multidisciplinary care planning 'item numbers' that provide financial incentives for general practices to employ practice nurses and to engage with other allied health providers. ${ }^{7}$ As a consequence, there has now been an exciting expansion in the educational and chronic disease management roles for practice nurses in Australia. Anecdotally, this role expansion has not widely embraced an "integrated platform of asthma education". ${ }^{2}$ Unless these financial incentives are established more broadly across primary care to actively foster team-based primary care with training and interest in comprehensive respiratory education, indirect BHR testing is unlikely to be widely implemented.

The increasing reality of limited health resources is forcing policy makers to demand sound economic data before they agree to any new financial reimbursement models. While cost-effectiveness analyses in primary care are more acceptable to clinicians, it is likely that these will have to be combined with cost-utility studies. ${ }^{8}$ GPs and other primary care clinicians fully understand that one of the confounders in understanding the real value of any new primary care respiratory intervention is patients' attendance for regular follow-up and ongoing medication compliance. As such, factoring in comprehensive measurement of patient preference in any economic analyses - using such tools as Discrete Choice Analyses (DCEs) - and the effects on such secondary outcomes as patient and carer satisfaction, will be integral to a fuller understanding of the economic value of such an innovation in primary care. ${ }^{9}$

The other hidden benefit in Turton et al.'s paper ${ }^{2}$ is their identification of the increasing value of point-of-care testing in fostering improved compliance and patient empowerment when it is integrated into daily clinical management in primary care. Their qualitative analyses revealed increased understanding of the chronic nature of asthma and improved involvement in goal-setting between patients and their primary care health professional. Interestingly, these qualitative data ${ }^{2}$ were gathered from BHR-positive patients, which casts some doubt on the external validity of the findings. As with many studies on asthma management in primary care, patients recruited into studies are already highly motivated and compliant. However, the value of point-of-care testing in primary care for fostering improved compliance and patient engagement cannot be underestimated: a study of point-of-care pathology testing versus testing provided by a pathology laboratory among 4,300 patients in rural and urban general practices across Australia found that the patients in the point-of-care testing arm were found to have the same or better medication compliance. ${ }^{10}$ Point-of-care testing will only increase in importance in primary care as we build and develop new 
partnerships with patients within an evolving team-based primary care structure.

Commissioned article; not externally peer-reviewed; accepted 20th December 2011; online 24th January 2012

(C) 2012 Primary Care Respiratory Society UK. All rights reserved http://dx.doi.org/10.4104/pcrj.2012.00005

Prim Care Respir J 2012; 21(1): 4-5

Conflicts of interest The author declares that he has no conflicts of interest in relation to this article.

\section{References}

1. Brannan J. Bronchial Hyperresponsiveness in the assessment of asthma control: airway hyperresponsiveness in asthma: its measurement and clinical significance. Chest 2010;138:11S-17S. http://dx.doi.org/10.1378/chest.10-0231

2. Turton J, Glasgow N, Brannan J. Feasibility and acceptability of using bronchial hyperresponsiveness to manage asthma in primary care: a pilot study. Prim Care Respir J 2012;21:28-34. http://dx.doi.org/10.4104/pcrj.2011.00079

3. Sont JK, Willems LN, Bel EH, van Krieken JH, Vandenbroucke JP, Sterk PJ. The AMPUL Study Group. Clinical control and histopathologic outcome of asthma when using airway hyperresponsiveness as an additional guide to long-term treatment. Am J Respir Crit Care Med 1999;159:1043-51.

4. Nuijsink M, Hop WC, Sterk PJ, Duiverman EJ, de Jongste JC. Long-term asthma treatment guided by airway hyperresponsiveness in children: a randomised controlled tria. Eur Respir J 2007;30(3):457-66. http://dx.doi.org/10.1183/09031936.00111806

5. Koenig SM, Murray JJ, Wolfe J, et al. Does measuring BHR add to guideline derived clinical measures in determining treatment for patients with persistent asthma? Respir Med 2008;102(5):665-73. http://dx.doi.org/10.1016/j.rmed.2007.12.023

6. Harris MF, Jayasinghe U, Taggart J, et al. Multi-disciplinary Team Care Plans in the management of patients with chronic disease in Australian general practice. Med $J$ Aust 2011;194(5):236-9.

7. Australian Government Department of Health and Ageing. MBS Primary Care Items. Chronic Disease Management (CDM) Medicare Item numbers http://www.health.gov.au/internet/main/publishing.nsf/content/mbsprimarycarechronicdiseasemanagement

8. Lin CCW, Hass M, Maher C, Machado L, van Tulder M. Cost-effectiveness of general practice care for low back pain. A systematic review. Eur Spine $J$ 2011;20:1012-23. http://dx.doi.org/10.1007/s00586-010-1675-4

9. Utens C, Gossens L, Smeenk L, et al. Effectiveness and cost-effectiveness of early assisted discharge for Chronic Obstructive Pulmonary Disease exacerbations: the design of a randomised controlled trial. BMC Public Health 2010;10:618. http://biomedcentral.com/1471-24581/10/618 http://dx.doi.org/10.1186/1471-2458-10-618

10. Gialamos A, Yelland L, Ryan $P$, et al. Does point of care testing lead to the same or better adherence to medication? A randomised control trial. The POCT in Genera Practice Trial in General Practice. Med J Aust 2009;191:487-91.

\section{Statins for COPD: a challenge to conventional beliefs?}

\section{See linked article by Lawes et al. on pg 35}

\section{*Mike Pearson ${ }^{a}$}

a Professor of Clinical Evaluation, University of Liverpool, UK

*Correspondence: Professor Mike Pearson, Clinical Sciences Building, University Hospital Aintree, Longmoor Lane, Liverpool, L9 7AL. Tel: +44 (0)151 5293857 Fax: +44 (0)151 5292873 E-mail: Michael.Pearson@liverpool.ac.uk

Every now and then a paper comes along which challenges the mould. Chronic obstructive pulmonary disease (COPD) is an airway condition that results from inhaling "dusts" (particularly the tar of cigarettes). So when a paper suggests that a drug affecting the vascular system may alter outcome, it questions our standard beliefs. In this issue of the $P C R J$ is a paper from Lawes et al. describing how statin use is associated with a $30 \%$ reduction in mortality in COPD over 4 years ${ }^{1}$ - an outcome effect that is an order of magnitude greater than the current mainstays of COPD drug treatments, inhaled steroids and long-acting bronchodilators. Indeed, these have disappointingly little or no effect on disease progression, although they do reduce exacerbation rates and improve quality of life. Only oxygen in the late hypoxic phase is disease modulating. So, even a hint of a disease modifying effect by statins has to provoke interest.

But could this paper be wrong? It is observational and thus cannot prove causality, and the authors are appropriately cautious in their inferences, especially with neither smoking nor lung function data.
However, it is a large study, subject inclusion was unselected - i.e. all acute COPD admissions in New Zealand, the prescribing of statins in primary care was independent of the admission and collected via an automated link, and death is an unequivocal outcome.

A further caveat is that absolute numbers of deaths in the two groups, i.e. those prescribed and not prescribed statins, were similar at 4 years. The differences are only seen when statistical controls are added. However, to argue that those with cardiovascular or diabetes co-morbidity should have similar outcomes to those without flies in the face of every known epidemiological study. An odds ratio showing $30 \%$ fewer deaths puts statin therapy into the same league as oxygen, but with applicability to a wider range of patients. The changes in the study are not explained by the expected lipid or cardiovascular effects, leading to the implication that statins may be altering the underlying COPD. Until this hypothesis is formally tested it remains exciting but unproven - but for a moment let us follow the potential trail of what it might mean.

Statins were introduced for their lipid-lowering effect which, by reducing deposition of cholesterol in arteries, is cardio-protective. But they also act on platelets and clotting ${ }^{2,3}$ and perhaps vasculitis. ${ }^{4}$ These non-lipid effects are debated ${ }^{5}$ but some are claiming that the effects of statins go beyond fat metabolism such that cardiovascular patients obtain a double benefit. ${ }^{6}$ Those arguing for a polypill' ${ }^{7}$ suggest that statin benefits are independent of cholesterol levels, which may imply that the primary effect of statins is on non-lipid mechanisms.

Yet there are no suggestions that statins act to reduce or block the effect of inflammation due to smoke deposits or other dusts in the lung, or on elastases. While it is possible that this is because no one has looked, the alternative is to ask more radical questions: 